\title{
Introduction
}

\section{Multi-Agent Systems for Energy Management}

\author{
Francesco Amigoni, Maria Gini* and Wolfgang Ketter \\ Guest Editors
}

The desire for efficient use of energy is growing throughout the world and is stimulating countless efforts toward more sustainable consumption of resources. The complexity of the existing energy infrastructure demands management, modelling, and control systems which are autonomous and distributed. Agentbased systems are naturally well-suited for such tasks.

This special issue presents a representative selection of current research efforts in agent-based energy management. Areas covered range from electricity markets to monitoring and control of deployed energy systems. In the former case, agents have a competitive nature and represent buyers and sellers; in the latter case, agents have a cooperative nature and represent devices or elements of the controlled systems. The wide range of applications suggests that agent-based systems can play a fundamental role in energy management and that their potential is still largely unexplored. The affiliations of the authors that contributed to this special issue show how agent-based systems are becoming common in engineering disciplines and not only in computer science.

The papers that compose this special issue are summarized in the following. Some papers address modelling aspects of energy management. The paper by Ceppi and Gatti presents a game theoretical approach to model wholesale electricity markets, where energy generators sell electricity to a public agency by means of a central auction. Problems arising in this area include the selection of the auction mechanism, the determination of the winner, and the computation of the optimal strategies for the generators.

Accurate models of electric power systems are required to predict faults and for efficient control. Moreover, the complexity of current power systems requires distributed modelling, as that presented by Prymek and Horak, which consists of a multi-agent system, called
Rice, used to model an electric power system for maintenance, control, and simulation purposes. The paper by Santofimia et al. addresses the important problem of power quality in electric networks and introduces a multi-agent system that, using a qualitative model of how the power grid behaves, not only gathers data, but is also able of anticipating and reacting to faults in the power grid.

The remaining papers are mainly concerned with control aspects of energy management. The interest for autonomous control of electric power systems is mainly triggered by the consideration that future electric power networks are expected to involve directly renewable energy resources that, due to their nature, are intermittent and variable. An efficient demand-side management, which reshapes the energy consumption pattern of the end-users during peak time or when consumption exceeds generation, is required to have efficient power networks. A survey of agent-based systems for matching energy supply and demand in the future power networks is presented by Badawy, Hirsch, and Albayrak in their paper.

An important aspect of controlling electric power systems is the management of emergency or fault situations. Sometimes, load shedding is the only option to maintain an electric power network alive under severe conditions and it is not surprising that a number of papers address agent-based solutions for load shedding. The paper by Muller and Onsongo addresses the decentralized control of electric power systems by using intelligent agents able to automatically determine the amount, location, and timing of load shedding in order to respond to emergency load conditions. Solanki, Solanki, and Schulz illustrate a load shedding method designed for electric distribution systems operating on land and on ships. The paper by Yu, Schulz, and Srivas- 
tava addresses the reconfiguration of the power distribution system in all electric ships, using a multi-agent system to change the topology of the power system when a fault happens and thus increasing the ship survivability, flexibility, and reliability. Finally, the paper by Cristaldi, Ponci, Riva, and Faifer considers electric power systems with limited power availability and proposes a multi-agent framework for load management in these systems. 